\title{
Translation of Time-Reversal Violation in the Neutral K-Meson System into a Table-Top Mechanical System
}

\author{
Andreas Reiser ${ }^{1}$, Klaus R. Schubert ${ }^{2}$, and Jürgen Stiewe ${ }^{1}$ \\ ${ }^{1}$ Kirchhoff-Institut für Physik, Universität Heidelberg, Heidelberg, Germany \\ ${ }^{2}$ Institut für Kern- und Teilchenphysik, Technische Universität Dresden, \\ Dresden, Germany \\ E-mail: Andreas.Reiser@kip.uni-heidelberg.de
}

\begin{abstract}
Weak interactions break time-reversal (T) symmetry in the two-state system of neutral $\mathrm{K}$ mesons. We present and discuss a two-state mechanical system, a Foucault-type pendulum on a rotating table, for a full representation of $\mathrm{K}^{0} \overline{\mathrm{K}}^{0}$ transitions by the pendulum motions including $\mathrm{T}$ violation. The pendulum moves with two different oscillation frequencies and two different magnetic dampings. Its equation of motion is identical with the differential equation for the real part of the CPT-symmetric $\mathrm{K}$-meson wave function. The pendulum is able to represent microscopic $\mathrm{CP}$ and $\mathrm{T}$ violation with CPT symmetry owing to the macroscopic Coriolis force which breaks the symmetry under reversal-of-motion. Video clips of the pendulum motions are shown as supplementary material.
\end{abstract}

\section{Introduction}

Symmetry considerations belong to the most fundamental and powerful tools in many fields of physics. Especially in particle physics, symmetries both under continuous and under discrete transformations play a very important role. Strong and electromagnetic interactions are symmetric under the three discrete transformations parity $\mathrm{P}(\vec{r} \leftrightarrow-\vec{r})$, charge conjugation $\mathrm{C}$ ( particle $\leftrightarrow$ antiparticle) and time reversal $\mathrm{T}$ ( $\mathrm{t} \leftrightarrow$-t and initial $\leftrightarrow$ final states). As observed in 1957 [1, 2], weak interactions break the discrete symmetries $\mathrm{P}$ and $\mathrm{C}$ maximally, but they are symmetric under the combination $\mathrm{CP}$ to a high level of accuracy. In 1964, a violation of CP symmetry at the level of $10^{-3}$ has been observed in transitions between $\mathrm{K}^{0}$ and $\overline{\mathrm{K}}^{0}$ mesons [3. Today we understand that these transitions and the small symmetry breaking therein are also produced by weak interactions. The Standard Theory of weak interactions is symmetric under the combination CPT. In this theory, $\mathrm{CP}$ violation goes along with $\mathrm{T}$ violation and is allowed to show up in any weak process involving three families of quarks [4. T violation and CPT symmetry in $\mathrm{K}^{0} \overline{\mathrm{K}}^{0}$ transitions has been confirmed by experiments [5, 6, 7], and no CPT-symmetry breaking process has been observed so far.

Using the present precise values of the $\mathrm{K}^{0} \overline{\mathrm{K}}^{0}$-transition parameters [8], figure 1 shows the time-dependent probabilities for (a) an initial $\mathrm{K}^{0}$ remaining a $\mathrm{K}^{0}$ and appearing as a $\overline{\mathrm{K}}^{0}$, and for (b) an initial $\overline{\mathrm{K}}^{0}$ remaining a $\overline{\mathrm{K}}^{0}$ and appearing as a $\mathrm{K}^{0}$. For better visibility, the size of the observed $\mathrm{T}$ violation has been increased by a factor of 10 . The equality of the two pobabilities $P\left[\mathrm{~K}^{0}(0) \rightarrow \mathrm{K}^{0}(t)\right]$ and $P\left[\overline{\mathrm{K}}^{0}(0) \rightarrow \overline{\mathrm{K}}^{0}(t)\right]$ means CPT symmetry, and the inequality $P\left[\overline{\mathrm{K}}^{0}(0) \rightarrow \mathrm{K}^{0}(t)\right]>P\left[\mathrm{~K}^{0}(0) \rightarrow \overline{\mathrm{K}}^{0}(t)\right]$ breaks $\mathrm{CP}$ as well as $\mathrm{T}$ symmetry. The two sums $P\left[\overline{\mathrm{K}}^{0}(0) \rightarrow \mathrm{K}^{0}(t)\right.$ or $\left.\overline{\mathrm{K}}^{0}(t)\right]$ and $P\left[\mathrm{~K}^{0}(0) \rightarrow \mathrm{K}^{0}(t)\right.$ or $\left.\overline{\mathrm{K}}^{0}(t)\right]$ in $(\mathrm{c})$ show that 
initial $\overline{\mathrm{K}}^{0}$ "live longer" than initial $\mathrm{K}^{0}$ mesons, i.e. have produced a smaller number of decays in the same interval of time.

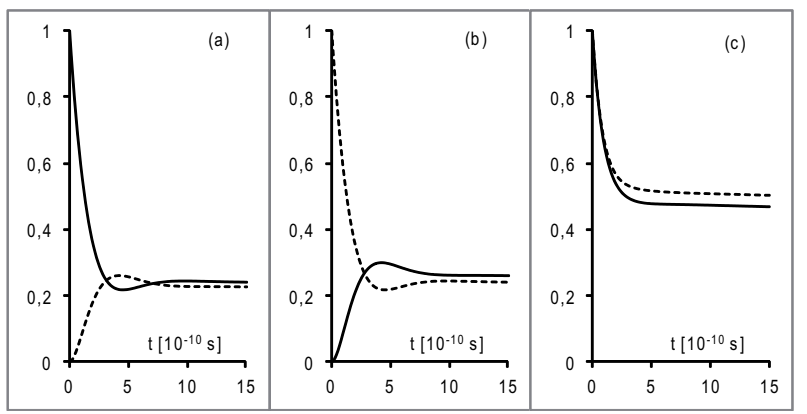

Figure 1: In (a) for a $\mathrm{K}^{0}$ at $\mathrm{t}=0$ and in (b) for a $\overline{\mathrm{K}}^{0}$ at $\mathrm{t}=0$, the solid curves show the $\mathrm{K}^{0}$ intensities and the dashed curves the $\overline{\mathrm{K}}^{0}$ intensities. In (c), the solid curve is the sum of the two curves in (a), and the dashed curve is the sum of the two curves in (b).

Several authors have proposed and constructed mechanical and electrical analoga of the $\mathrm{K}^{0} \overline{\mathrm{K}}^{0}$ system starting, to our knowledge, with Winstein in 1987. He constructed and presented a pair of coupled pendula swinging in one dimension. In the writeup of his presentation [9], Winstein proved that his analogon is principally not able to show $\mathrm{T}$ violation because of the time-reversal symmetry of the equations of motion for the pendulum pair. In 1996, Rosner [10] proposed that the Coriolis force can be used for demonstrating $\mathrm{T}$ violation in $\mathrm{K}^{0} \overline{\mathrm{K}}^{0}$ transitions with one pendulum swinging in two dimensions on a turntable. Kostelecki and Roberts [11] discussed this further and also proposed to use the T violating properties of a gyrator in a setup with two coupled electrical circuits. The latter proposal has recently been discussed again by Caruso et al. [12. We are not aware that one of these $\mathrm{T}$ violating proposals has been built so far and we therefore present in this article the construction of a table-top Foucault pendulum.

Our setup follows the suggestion of Rosner and Slezak [13] with anisotropic oscillations and dampings of the pendulum. In chapter II we describe the construction, in chapter III we present the equation of motion and its solutions. In chapter IV we show that the real parts of the Schrödinger-equation solutions for the $\mathrm{K}^{0} \overline{\mathrm{K}}^{0}$ system obey a second-order differential equation which is formally identical with that for the coordinates of the pendulum. Therefore, as shown previously for the pair of coupled pendula [14] and by Caruso et al. 12, the table-top Foucault pendulum is not only demonstration and illustration of the $\mathrm{K}^{0} \overline{\mathrm{K}}^{0}$ system, but also isomorphic mapping, "translation" or equivalence in a stricter sense. The effect of the macroscopically T-violating Coriolis force is able to represent the microscopic $\mathrm{T}$ violation in $\mathrm{K}^{0} \overline{\mathrm{K}}^{0}$ transitions. In chapter $\mathrm{V}$, after discussing some control measurements, we present the motions of the pendulum and add some extensions. Six video clips of the motions are added to the article as supplementary material 11 .

\footnotetext{
${ }^{1}$ http://www.kip.uni-heidelberg.de/user/reiser/video
} 


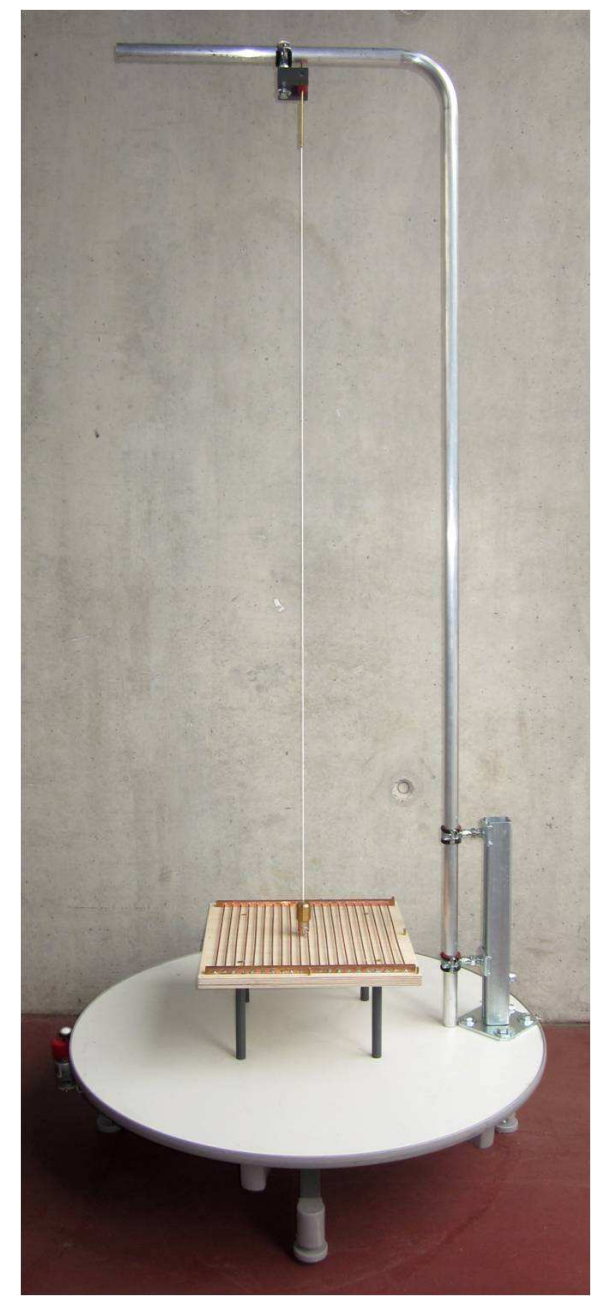

Figure 2: Photograph of the pendulum setup

\section{Apparatus - A Foucault Pendulum on a Turntable}

The Foucault pendulum for representing $\mathrm{T}$ violation is constructed as a portable system; figure 2 shows a photograph and figure 3 a drawing of its main components. The rotating frame of reference is a wooden circular plate with a diameter of $1 \mathrm{~m}$. Since there is no preferred direction in the rotating plane, we choose an arbitrary direction as $\mathrm{x}$-axis representing the $\mathrm{K}^{0}$ state. The perpendicular direction y represents the $\overline{\mathrm{K}}^{0}$ state. The axial vector $\Omega \vec{e}_{z}$ describes the rotation, and $\vec{e}_{x}, \vec{e}_{y}, \vec{e}_{z}$ form a right-handed orthogonal coordinate system. The turntable is mounted on a steel construction which has four adjustable feet at the lower part and a ball-borne axis carrying four upper arms. The wooden plate is fixed on these arms. We use an electric gear dc motor to drive the turntable. The motor is mounted at one of the lower arms. Its axis carries a rubber wheel. The complete drive unit is pressed to the circumference of the wooden table using a spring. This ensures a good frictional contact even if the wooden plate is not perfectly circular. The accessible 
rotation speed of the turntable ranges between 0 and $0.1 \mathrm{rpm}$ and can be adjusted by setting the appropriate drive voltage for the motor. Fixed at an aluminium stand mounted onto the turntable, a non-twisting nylon thread of length $\ell=1.35 \mathrm{~m}$ carries the pendulum bob. The upper part of the thread can only swing back and forth in $(x+y)$ direction enforced by a hinge and a tube of length $\delta \ell=10 \mathrm{~cm}$ fixed to the hinge. The lower part of the thread can swing freely in any direction. For motions in the $(\mathrm{x}-\mathrm{y})$ direction we have $\Omega_{-}=\sqrt{g /(\ell-\delta \ell)}$, in the $(\mathrm{x}+\mathrm{y})$ direction $\Omega_{+}=\sqrt{g / \ell}$. The pendulum bob is made of a cylindrical brass weight, $30 \mathrm{~mm}$ long and $30 \mathrm{~mm}$ in diameter. The pendulum thread is fixed using a centric bore of $1 \mathrm{~mm}$ diameter in the brass weight. One ring-shaped permanent dipolar magnet with an outer diameter of $20 \mathrm{~mm}$, inner diameter of $10 \mathrm{~mm}$ and height $10 \mathrm{~mm}$ is glued to the brass weight using an epoxy resin. The material is an alloy (N45) made of $\mathrm{NdFeB}$, with a maximum field of approximately $1.37 \mathrm{~T}$ at the surface. The lower end of the brass weight is machined to fit tightly into the bore of the magnet. A second N45 magnet, disc-shaped with an diameter of $20 \mathrm{~mm}$ and height $10 \mathrm{~mm}$ forms the closure of the pendulum bob and enlarges the magnetic field. The dipole moment of each magnet is oriented along the cylinder axis.
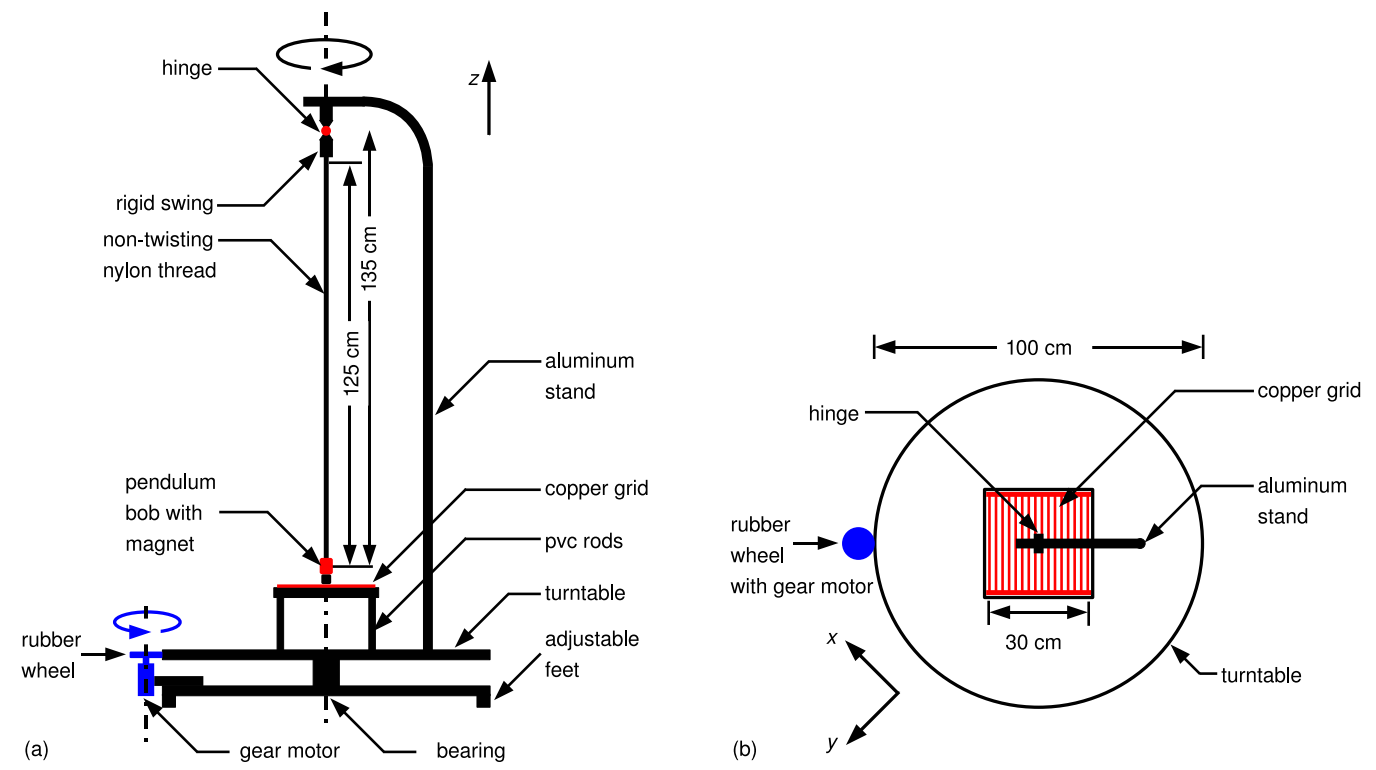

Figure 3: Side (a) and top (b) view of the complete pendulum setup

Supported by four $15 \mathrm{~cm}$ long pvc rods, a square wooden plate of $33 \mathrm{~cm}$ edge length is used to carry the damping device. This small table is screwed at the turntable centrically. The damping device consists of a copper-rod grid in which eddy currents are induced by the magnetic bob. The copper rods are $30 \mathrm{~cm}$ long and have a diameter of $3 \mathrm{~mm}$. The spacing between two copper rods is $1.5 \mathrm{~cm}$. The copper grid is closed at both ends via soft-soldering the copper rods together into a massive copper bar $31 \mathrm{~cm}$ long and $10 \times 10 \mathrm{~mm}$ in cross section. This low-ohmic electrical shortcut bar is necessary for an effective eddy current brake. The length of the pvc rods was chosen in order to avoid magnetic disturbance 
of the pendulum bob originating from the steel structure of the turntable. All materials close to the magnetic pendulum bob are non-magnetic in common sense (wood, copper, brass, pvc). The copper grid lies in the $x y$ plane and is aligned paralled to the $(x-y)$ direction. The magnetic pendulum bob is swinging above this grid with a minimum distance of $5 \mathrm{~mm}$. The damping in $(x-y)$ direction is small whereas it is large in the $(x+y)$ direction. To explain the damping mechanism we consider the magnetic flux caused by the magnetic pendulum bob. When moving the magnet along the rods the flux change in the shortcut loops of the grid is practically vanishing and no damping force is present. A movement perpendicular to the copper rods leads to the maximum flux change within the loops causing a damping force owing to Lenz' rule. With this setup, the damping force is approximately proportional to the bob velocity.

\section{Equation of Motion and its Solutions}

The damped harmonic oscillator with a period $T=2 \pi / \omega$ has the equation of motion

$$
\ddot{x}=-\omega^{2} x-\Gamma \dot{x}
$$

where $1 / \Gamma$ is the time in which its energy decreases by $1 /$ e and $2 / \Gamma$ the same for the amplitude. The solution of the equation is

$$
x(t)=A \mathrm{e}^{-\Gamma t / 2} \cos \left(\omega_{0} t-B\right)
$$

with two free parameters $A$ and $B$, and $\omega_{0}=\sqrt{\omega^{2}-\Gamma^{2} / 4}$. With $\Omega=0$, two coordinates of the pendulum bob, $x-y$ and $x+y$, obey equation (11) if we neglect the z coordinate and restrict the motions to small deflection angles:

$$
\begin{aligned}
& \ddot{x}-\ddot{y}=-\omega_{-}^{2}(x-y)-\Gamma_{-}(\dot{x}-\dot{y}), \\
& \ddot{x}+\ddot{y}=-\omega_{+}^{2}(x+y)-\Gamma_{+}(\dot{x}+\dot{y}) .
\end{aligned}
$$

The two equations are uncoupled. If we rotate the turntable, we have to add the Coriois term $+2 \Omega(\dot{x}+\dot{y})$ to the first equation and $-2 \Omega(\dot{x}-\dot{y})$ to the second one, and we obtain a pair of coupled equations. The signs of the Coriolis terms follow from the righthandedness of the system $x-y, x+y, \Omega$. In $x$ and $y$, the coupled equation of motion is

$$
\left(\begin{array}{c}
\ddot{x} \\
\ddot{y}
\end{array}\right)=-\left(\begin{array}{cc}
\omega_{0}^{2} & \omega_{0} \cdot \Delta \omega \\
\omega_{0} \cdot \Delta \omega & \omega_{0}^{2}
\end{array}\right)\left(\begin{array}{l}
x \\
y
\end{array}\right)-\left(\begin{array}{cc}
\Gamma & \Delta \Gamma / 2-2 \Omega \\
\Delta \Gamma / 2+2 \Omega & \Gamma
\end{array}\right)\left(\begin{array}{l}
\dot{x} \\
\dot{y}
\end{array}\right),
$$

where we have introduced the abbreviations

$$
\omega_{0}=\left(\omega_{+}+\omega_{-}\right) / 2, \quad \Delta \omega=\omega_{+}-\omega_{-}, \quad \Gamma=\left(\Gamma_{+}+\Gamma_{-}\right) / 2, \quad \Delta \Gamma=\Gamma_{+}-\Gamma_{-}
$$

and have used the approximation $|\Delta \omega| \ll \omega_{0}$. Without further approximation, equation (44) has no solutions of the form in equation (2). With the approximation $|\Omega| \ll|\Delta \Gamma|$, however, the two eigensolutions are of this form:

$$
\left(\begin{array}{l}
x \\
y
\end{array}\right)_{S}=A\left(\begin{array}{l}
p \\
q
\end{array}\right) \mathrm{e}^{-\Gamma_{S} t / 2} \cos \left(\omega_{S} t-B\right), \quad\left(\begin{array}{l}
x \\
y
\end{array}\right)_{L}=C\left(\begin{array}{c}
p \\
-q
\end{array}\right) \mathrm{e}^{-\Gamma_{L} t / 2} \cos \left(\omega_{L} t-D\right),
$$


with

$$
\omega_{S}=\omega_{+}, \quad \omega_{L}=\omega_{-}, \quad \Gamma_{S}=\Gamma_{+}, \quad \Gamma_{L}=\Gamma_{-}, p / q=1-4 \Omega / \Delta \Gamma,
$$

and $p^{2}+q^{2}=1$. In our apparatus, we have chosen $\Gamma_{+}>\Gamma_{-}$, i. e. we have $\Delta \Gamma>0$ and the index $S$ and $L$ denotes, as in the Kaon system, the short- and long-living eigenstate, respectively. For further equivalence with the Kaon system, our demonstration has to use $\Delta \omega<0$ since the mass difference between $\mathrm{K}^{0}{ }_{S}$ and $\mathrm{K}^{0}{ }_{L}$ is negative, and we have to turn the table clockwise $(\Omega<0)$ since $p / q=1+2 \operatorname{Re}\left(\epsilon_{\mathrm{K}}\right)>1$.

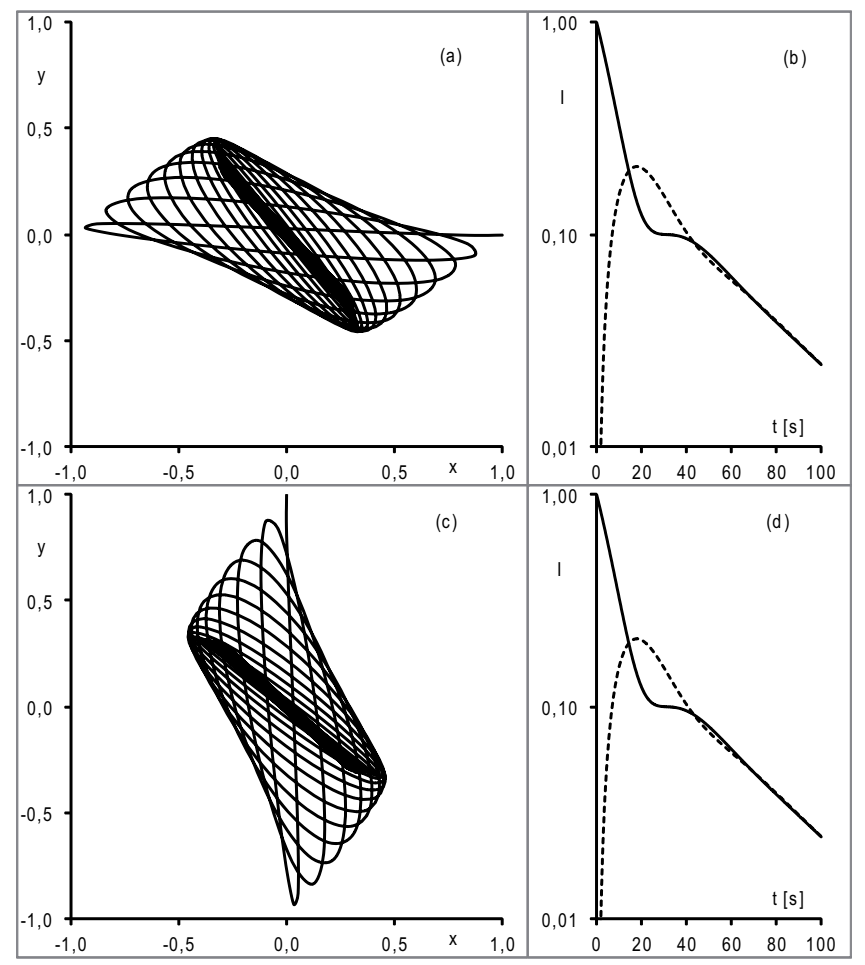

Figure 4: (a) Pendulum orbit with $\Omega=0$ when starting the motion at $(x, y)=(1,0)$ for the first $100 \mathrm{~s}$. (b) The solid curve shows the intensity $<2 x^{2}>$, the dashed curve $<2 y^{2}>$. (c) Orbit with $\Omega=0$ when starting at $(x, y)=(0,1)$. (d) Intensities $<2 y^{2}>$ (solid curve) and $<2 x^{2}>$ (dashed curve) for the second motion; both curves are identical with those in (b). The parameters for the calculation are taken from the demonstration setup as given in chapter V. The asymptotic motions in (a) and (c) follow a straight line along the $(x-y)$ direction.

The most general solution of equation (4), the sum of the two eigenfunctions in equation (6), has four free parameters since the equation of motion is of second order. The four parameters are fixed by the initial conditions $x(0), y(0), \dot{x}(0)$ and $\dot{y}(0)$. The Schrödinger equation for the Kaon system is only of first order, see chapter IV. Its most general solution contains only two free parameters to be fixed by $\psi_{1}(0)$ and $\psi_{2}(0)$. In the translation of the Kaon system into the pendulum, this means that we can only fix $A$ and $C$ by $x(0)$ and $y(0)$, and the non-adjustable decay rates $\dot{x}(0)$ and $\dot{y}(0)$ lead to $B=D=0$. 
With $\Omega=0$, figure 4 shows the orbits $(x, y)$ and intensities for two special solutions, with $(x, y)=(1,0)$ and $(x, y)=(0,1)$ at $t=0$; both are presented here with $B=D=0$. Since it is hard to implement $(\dot{x}, \dot{y})=(-\Gamma / 2,0)$ or $(0,-\Gamma / 2)$ at $t=0$ in the demonstration of the pendulum, we have checked that the $B$ and $D$ values originating from $(\dot{x}, \dot{y})=(0,0)$ lead to orbits which show no noticeable difference from those with $B=D=0$. In the first 20 seconds the orbit in figure 4(a), i.e. the translation of the evolution of a pure $\mathrm{K}^{0}$ at $t=0$, shows a counterclockwise elliptical motion. Hereby the ellipse axes rotate clockwise. In figure $4(\mathrm{c})$, showing the evolution of a pure $\overline{\mathrm{K}}^{0}$, the two rotating senses are inverted. This difference is not seen in the intensities $\left\langle 2 x^{2}\right\rangle$ and $\left\langle 2 y^{2}\right\rangle$, the intensities are equal. Because of the strong damping of the $(x+y)$ component of the motion, both orbits end in the less-damped linear motion in the $(x-y)$ direction. In this asymptotic motion, there are equal amplitudes in $x$ and $y$, and the remaining motion energy is the same for both orbits. There is a complete symmetry between $x$ and $y$.

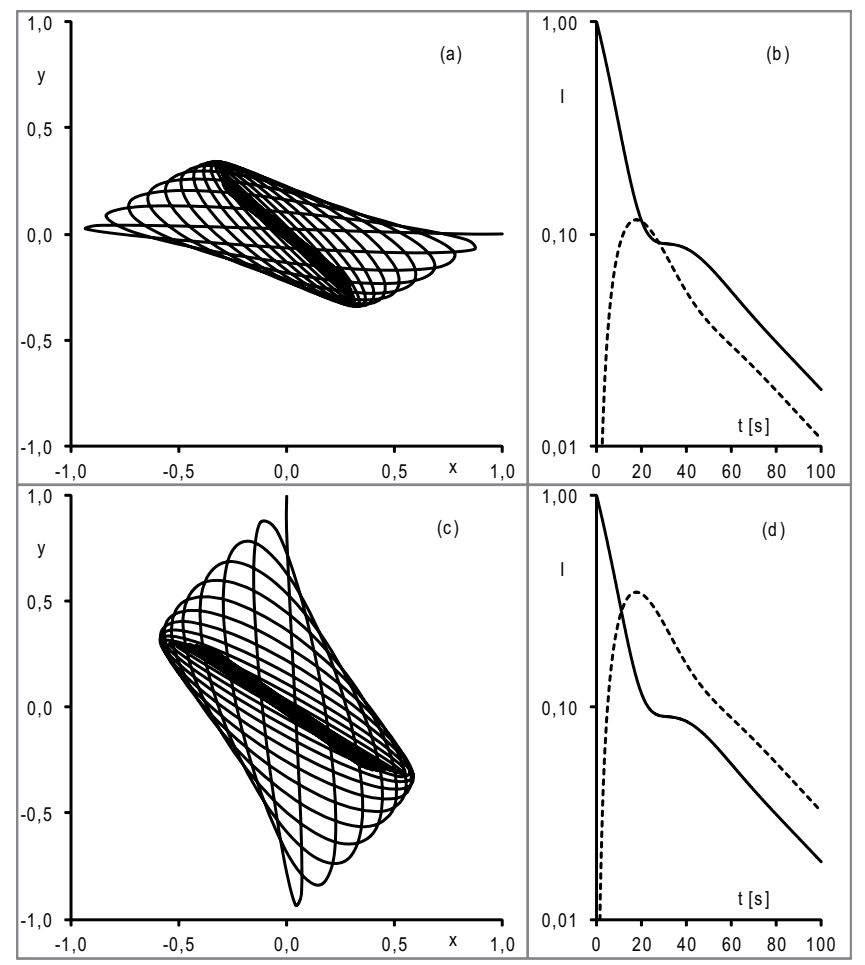

Figure 5: (a) Pendulum orbit with $\Omega=-0.01 / s$ when starting the motion at $(x, y)=(1,0)$ for the first $100 \mathrm{~s}$. (b) The solid curve shows the intensity $\left\langle 2 x^{2}\right\rangle$, the dashed curve $<2 y^{2}>$. (c) Orbit with $\Omega=-0.01 / s$ when starting at $(x, y)=(0,1)$. (d) Intensities $\left.<2 y^{2}\right\rangle$ (solid curve) and $\left\langle 2 x^{2}\right\rangle$ (dashed curve) for the second motion. The asymptotic motions in (a) and (c) follow a straight line inclined by 7.6 degrees from the $(x-y)$ direction.

Figure 5 shows the orbits and intensities for a clockwise table rotation with $\Omega=-0.01 / \mathrm{s}$. 
The transition features $x \rightarrow y$ and $y \rightarrow x$ are very much the same as for $\Omega=0$. The small observed difference is created by the T-violating Coriolis force. After damping the $\mathrm{S}$ component of the motion, the asymptotic L motion has two pronounced features in breaking the symmetry between $x$ and $y$ : First, the $x$ component is larger than the $y$ component for both initial conditions, and second, the remaining motion energy at any time is larger when starting with $(x, y)=(0,1)$ than that for starting with $(x, y)=(1,0)$. Both features originate from the time-reversal violation $x^{2}(t) / y^{2}(0)>y^{2}(t) / x^{2}(0)$ created by the Coriolis force.

\section{Translating the Schrödinger Equation of the $\mathrm{K}^{0} \overline{\mathrm{K}}^{0}$ System}

The time evolution of the wave function $\Psi=\psi_{1} \mathrm{~K}^{0}+\psi_{2} \overline{\mathrm{K}}^{0}$ for the $\mathrm{K}^{0} \overline{\mathrm{K}}^{0}$ system with CPT symmetry is given by the Schrödinger equation

$$
\mathrm{i} \frac{\partial}{\partial t}\left(\begin{array}{c}
\psi_{1} \\
\psi_{2}
\end{array}\right)=\left[\left(\begin{array}{cc}
m & m_{12} \\
m_{12}^{*} & m
\end{array}\right)-\frac{\mathrm{i}}{2}\left(\begin{array}{cc}
\Gamma & \Gamma_{12} \\
\Gamma_{12}^{*} & \Gamma
\end{array}\right)\right]\left(\begin{array}{c}
\psi_{1} \\
\psi_{2}
\end{array}\right)
$$

with five observable real parameters, $m, \Gamma,\left|m_{12}\right|$, Re $\left(\Gamma_{12} / m_{12}\right)$ and $\operatorname{Im}\left(\Gamma_{12} / m_{12}\right)$. $\mathrm{T}$ is violated iff $\operatorname{Im}\left(\Gamma_{12} / m_{12}\right) \neq 0$. The two fundamental solutions are

$$
\left(\begin{array}{l}
\psi_{1} \\
\psi_{2}
\end{array}\right)_{S}=\left(\begin{array}{c}
p \\
q
\end{array}\right) \cdot \mathrm{e}^{-\mathrm{i} m_{S} t} \mathrm{e}^{-\Gamma_{S} t / 2}, \quad\left(\begin{array}{c}
\psi_{1} \\
\psi_{2}
\end{array}\right)_{L}=\left(\begin{array}{c}
p \\
-q
\end{array}\right) \cdot \mathrm{e}^{-\mathrm{i} m_{L} t} \mathrm{e}^{-\Gamma_{L} t / 2},
$$

where the five observables $m_{S}, \Gamma_{S}, m_{L}, \Gamma_{L}$ and $|p / q|$ follow unambiguously from the five parameters of the Schrödinger equation [15. The values of $p$ and $q$ can be chosen to be real, leading to

$$
\left(\begin{array}{l}
\Re_{1} \\
\Re_{2}
\end{array}\right)_{S}=\left(\begin{array}{c}
p \\
q
\end{array}\right) \cdot \mathrm{e}^{-\Gamma_{S} t / 2} \cos \left(m_{S} t\right), \quad\left(\begin{array}{c}
\Re_{1} \\
\Re_{2}
\end{array}\right)_{L}=\left(\begin{array}{c}
p \\
-q
\end{array}\right) \cdot \mathrm{e}^{-\Gamma_{L} t / 2} \cos \left(m_{L} t\right) .
$$

The real parts $\Re_{1}$ and $\Re_{2}$ of $\psi_{1}$ and $\psi_{2}$, respecively, obey the second-order linear differential equation [14]

$$
\begin{array}{r}
\left(\begin{array}{c}
\ddot{\Re}_{1} \\
\ddot{\Re}_{2}
\end{array}\right)=-\left(\begin{array}{rr}
\frac{m_{S}^{2}+m_{L}^{2}}{2}+\frac{\Gamma_{S}^{2}+\Gamma_{L}^{2}}{8} & \frac{p}{q} \cdot\left(\frac{m_{S}^{2}-m_{L}^{2}}{2}+\frac{\Gamma_{S}^{2}-\Gamma_{L}^{2}}{8}\right) \\
\frac{q}{p} \cdot\left(\frac{m_{S}^{2}-m_{L}^{2}}{2}+\frac{\Gamma_{S}^{2}-\Gamma_{L}^{2}}{8}\right) & \frac{m_{S}^{2}+m_{L}^{2}}{2}+\frac{\Gamma_{S}^{2}+\Gamma_{L}^{2}}{8}
\end{array}\right)\left(\begin{array}{l}
\Re_{1} \\
\Re_{2}
\end{array}\right) \\
-\left(\begin{array}{cc}
\frac{\Gamma_{S}+\Gamma_{L}}{2} & \frac{p}{q} \cdot \frac{\Gamma_{S}-\Gamma_{L}}{2} \\
\frac{q}{p} \cdot \frac{\Gamma_{S}-\Gamma_{L}}{2} & \frac{\Gamma_{S}+\Gamma_{L}}{2}
\end{array}\right)\left(\begin{array}{l}
\dot{\Re}_{1} \\
\dot{\Re}_{2}
\end{array}\right) .
\end{array}
$$

In the $\mathrm{K}^{0} \overline{\mathrm{K}}^{0}$ system, the phase of $\Gamma_{12} / m_{12}$ is small and negative. Therefore, $p / q-1$ is small and positive, and we have in good approximation $\left(m_{S}+m_{L}\right) / 2=m, m_{S}-m_{L}=$ $\Delta m=-2\left|m_{12}\right|,\left(\Gamma_{S}+\Gamma_{L}\right) / 2=\Gamma$ and $\Gamma_{S}-\Gamma_{L}=\Delta \Gamma=2\left|\Gamma_{12}\right|$. In addition, $|\Delta m|$ and $\Gamma$ are much smaller than $m$. Neglecting all terms that are small in second order, equation 11 becomes

$$
\left(\begin{array}{l}
\ddot{\Re}_{1} \\
\ddot{\Re}_{2}
\end{array}\right)=-\left(\begin{array}{cc}
m^{2} & m \cdot \Delta m \\
m \cdot \Delta m & m
\end{array}\right)\left(\begin{array}{l}
\Re_{1} \\
\Re_{2}
\end{array}\right)-\left(\begin{array}{cc}
\Gamma & \frac{p}{q} \cdot \frac{\Delta \Gamma}{2} \\
\frac{q}{p} \cdot \frac{\Delta \Gamma}{2} & \Gamma
\end{array}\right)\left(\begin{array}{l}
\dot{\Re}_{1} \\
\dot{\Re}_{2}
\end{array}\right) .
$$


With the substitution

$$
\frac{p}{q}=1-\frac{4 \Omega}{\Delta \Gamma},
$$

and $|\Omega| \ll|\Delta \Gamma|$ this is the equation of motion for our Foucault pendulum with two different damping rates and two different oscillation frequencies:

$$
\left(\begin{array}{c}
\ddot{x} \\
\ddot{y}
\end{array}\right)=-\left(\begin{array}{cc}
\omega_{0}^{2} & \omega_{0} \cdot \Delta \omega \\
\omega_{0} \cdot \Delta \omega & \omega_{0}^{2}
\end{array}\right)\left(\begin{array}{l}
x \\
y
\end{array}\right)-\left(\begin{array}{cc}
\Gamma & \Delta \Gamma / 2-2 \Omega \\
\Delta \Gamma / 2+2 \Omega & \Gamma
\end{array}\right)\left(\begin{array}{c}
\dot{x} \\
\dot{y}
\end{array}\right) .
$$

Since the necessary approximations $|\Delta m| \ll m, \Gamma \ll m$ and $|p / q-1| \ll 1$ are well fulfilled, our pendulum is a good representation of the $\mathrm{K}^{0} \overline{\mathrm{K}}^{0}$ system. The time-averaged amplitude-squares of the real parts in equation 12 are equal to the modulus-squares in equation 8 ,

$$
\left\langle 2 x^{2}\right\rangle_{T}=\left|\psi_{1}\right|^{2},\left\langle 2 y^{2}\right\rangle_{T}=\left|\psi_{2}\right|^{2},
$$

with averaging-intervals $T$ which are much larger than $1 / \omega_{0}$ and much smaller than $1 / \Gamma$ and $|1 / \Delta \omega|$. Therefore, the squares of the amplitudes $x$ and $y$ of the pendulum represent the $\mathrm{K}^{0}$ and $\overline{\mathrm{K}}^{0}$ intensities, respectively, in the evolution of $\Psi$ [14].

\section{Controls, Demonstrations, Extensions}

First, we controlled the bob orbits without rotating the table and with only one oscillation frequency by blocking the swing hinge. Starting the pendulum in the $(\mathrm{x}+\mathrm{y})$ direction leads to a quickly damped linear oscillation as shown in video 1 and starting it in the direction $(\mathrm{x}-\mathrm{y})$ to the much less damped linear oscillation in video 2 . We measured the decay times $2 / \Gamma_{+}=11.5 \mathrm{~s}$ and $2 / \Gamma_{-}=86 \mathrm{~s}$, defined by the time in which the amplitudes decrease to a level of $1 / \mathrm{e}$.

Second, we add the feature with two different pendulum lengths. This leaves the two motions in videos 1 and 2 essentially unchanged, but the oscillaion frequency in (x-y) is slightly higher than in $(\mathrm{x}+\mathrm{y})$ because of $\Delta \omega / \Delta \Gamma<0$. With this setup, i. e. still with $\Omega=0$, we next controlled the bob orbits when starting with $(x, y, \dot{x}, \dot{y})=\left(R_{0}, 0,0,0\right)$ and with $(x, y, \dot{x}, \dot{y})=\left(0, R_{0}, 0,0\right)$. These motions are shown in videos 3 and 4 , respectively. Very soon after the start in video 3 , we see a counterclockwise rotating elliptical motion with the ellipse axes rotating clockwise. In video 4 , the motion starts with clockwise rotating ellipses, and the axes rotate counterclockwise. In both motions, the ellipses become first more circle-like and then slimmer again. After about $10 \mathrm{~s}$, the originally counterclockwise motion changes into a slim clockwise ellipse, and vice versa in video 4 . After about $60 \mathrm{~s}$, both motions become linear in the (x-y) direction, i. e. with equal coordinates $\mathrm{x}$ and $\mathrm{y}$. The motions show energy transitions from the $\mathrm{x}$ direction to the $\mathrm{y}$ direction in video 3 and from $\mathrm{y}$ to $\mathrm{x}$ in video 4 . Both agree with the calculated orbits in figure 4, and both are the translation of transitions from $\mathrm{K}^{0}$ to $\overline{\mathrm{K}}^{0}$ and from $\overline{\mathrm{K}}^{0}$ to $\mathrm{K}^{0}$, respectively, in the $\mathrm{CP}$ - and $\mathrm{T}$-conserving approximation. CP symmetry is seen in the equality of $\mathrm{x}$ and $\mathrm{y}$ at large times.

The complete setup with a clockwise table rotation $(\Omega=-0,01 / \mathrm{s})$ is then demonstrated in videos 5 and 6 , starting with the $\mathrm{x}$ motion and with the y motion, respectively. Both motions agree with the calculated results in figure 5. For early times, they resemble very 
much the T-symmetric motions with $\Omega=0$. But already at $\mathrm{t}$ around $10 \mathrm{~s}$, we start to see different motion patterns. The change of rotation sense is more pronounced in video 6 than in video 5. At later times, both motions end to be linear oscillations in the expected direction which is different from $\mathrm{x}=\mathrm{y}$. The $\mathrm{x}$ component is larger than the $\mathrm{y}$ component in both motions, as given by the ratio of $\Omega / \Delta \Gamma$ in equation 7 . This is the translation of $\mathrm{CP}$ and $\mathrm{T}$ violation in transitions between $\mathrm{K}^{0}$ and $\overline{\mathrm{K}}^{0}$. Also the message of figure 1 (c) that an initial $\overline{\mathrm{K}}^{0}$ "lives longer" than an initial $\mathrm{K}^{0}$ is clearly visible when comparing the amplitudes of the oscillations in the two motions at the same time t greater than $50 \mathrm{~s}$.

The pair of coupled pendula [14] is able to demonstrate $\mathrm{CP}$ violation in $\mathrm{K}^{0} \overline{\mathrm{K}}^{0}$ transitions, but in the translation of a quantum-mechanical evolution which is T-symmetric and CPT-violating. The Foucault pendulum on a rotating table, however, demonstrates CP violation in these transitions together with $\mathrm{T}$ violation and CPT symmetry. All aspects of the $\mathrm{K}^{0} \overline{\mathrm{K}}^{0}$-transition properties in figure 1 are properly demonstrated in the Foucaultpendulum motions.

With only minor modifications, we could also demonstrate $\mathrm{D}^{0} \overline{\mathrm{D}}^{0}, \mathrm{~B}^{0} \overline{\mathrm{B}}^{0}$ and $\mathrm{B}_{\mathrm{s}} \overline{\mathrm{B}}_{\mathrm{s}}$ transitions with our Foucault pendulum. At the present level of experimental knowledge, CP and $\mathrm{T}$ violation do not play an important role in these transitions. Since all three values of $p / q$ are compatible with 1 , we do not need a turntable. In addition, the isotropic fraction of the damping is large in all three systems, $\Gamma \gg|\Delta \Gamma|$, in contrast to the K-meson system. We could replace the eddy-current grid by a solid copper plate for the two B-meson sytems, and by a grid with nearly quadratic cells for the $\mathrm{D}^{0} \overline{\mathrm{D}}^{0}$ system. The three very different $\Delta m$ values could be translated into rigid swings at the upper end of the thread with three different lengths $\delta \ell$.

\section{Summary}

Following a proposal of Rosner and Slezak [13] in 2001, we have built a Foucault pendulum on a turntable with two different oscillation frequencies and two different dampings. The equations of motion for the two coordinates of this system are the real-part translations of the Schrödinger equation for the two components of the neutral K-meson system. T violation in the $\mathrm{K}$ system is translated into noninvariance under reversal-of-motion created by the Coriolis force. The consequences of both CPT symmetry and T violation, i. e. equal probabilities for a $\mathrm{K}^{0}$ at $t=0$ remaining a $\mathrm{K}^{0}$ at any $t>0$ and for a $\overline{\mathrm{K}}^{0}$ at $t=0$ remaining a $\overline{\mathrm{K}}^{0}$ at the same later time, and different probabilities for the transition probabilities for $\mathrm{K}^{0} \rightarrow \overline{\mathrm{K}}^{0}$ and $\overline{\mathrm{K}}^{0} \rightarrow \mathrm{K}^{0}$, become visible in the motions of the Foucault pendulum.

\section{Acknowledgements}

We thank Christian Enss and Karlheinz Meier for their encouragement and support as well as Siegfried Spiegel in the mechanical workshop of the Kirchhoff Institute for his valuable help in assembling and testing our system. 


\section{References}

[1] C. S. Wu et al., Phys. Rev. 105 , 1413 (1957)

[2] R. L. Garwin et al., Phys. Rev. 105, 1415 (1957)

[3] J. H. Christenson et al., Phys. Rev. Lett. 13, 138 (1964)

[4] M. Kobayashi and K. Maskawa, Progr. Theor. Phys. 49, 282 (1972)

[5] K. R. Schubert et al., Phys. Lett. 31 B, 662 (1970)

[6] A. Angelopoulos et al. (CPLEAR), Phys. Lett. B 444, 43 (1998)

[7] M. Fidecaro and H.-J. Gerber, Rep. Prog. Phys. 69, 1713 (2006)

[8] K. Nakamura et al. (Particle Data Group), J. Phys. G 37, 075021 (2010)

[9] B. Winstein, "CP Violation", Festi-Val - Festschrift for Val Telegdi, edited by K. Winter, Elsevier Amsterdam, p. 245 (1988)

[10] J. L. Rosner, Am. J. Phys. 64, 982 (1996)

[11] V. A. Kostelecky and A. Roberts, Phys. Rev. D 63, 096002 (2001)

[12] M. Caruso, H. Fanchiotti, and C. A. Garcia Canal, Annals of Physics 326, 2717 (2011)

[13] J. L. Rosner and S. A. Slezak, Am. J. Phys. 69, 44 (2001)

[14] K. R. Schubert and J. Stiewe, J. Phys. G: Nucl. Part. Phys. 39, 033101 (2012)

[15] G. C. Branco, L. Lavoura, and J. P. Silva, "CP Violation", Oxford University Press (1999) 